\title{
STUDY ON CARDIAC INVOLVEMENT IN SYSTEMIC SCLEROSIS
}

\author{
Mayilsamy Saravanan 1 , Murugesan Nagaventhan², Periyasamy Arivudainambi3, Murugan Subramani ${ }^{4}$
}

${ }_{1}^{1}$ Assistant Professor, Department of Rheumatology, KAPV Government Medical College, Tiruchy, Tamilnadu, India. ${ }^{2}$ Assistant Professor, Department of Gastroenterology, KAPV Government Medical College, Tiruchy, Tamilnadu, India. ${ }^{3}$ Assistant Professor, Department of Thoracic Medicine, KAPV Government Medical College, Tiruchy, Tamilnadu, India. ${ }_{4}^{4}$ Assistant Professor, Department of Internal Medicine, KAPV Government Medical College, Tiruchy, Tamilnadu, India. ABSTRACT

\section{BACKGROUND}

Systemic sclerosis is a generalised disorder of connective tissue characterised by diffuse vascular lesions, fibrosis of skin and major organ systems involvement including cardiac, pulmonary and gastrointestinal system. It also involves cardiac valves, pericardium, myocardium and coronary vessels.

The aim of this descriptive study is to assess the cardiac involvement in patients with systemic sclerosis.

\section{MATERIALS AND METHODS}

It was a descriptive study. 25 patients of systemic sclerosis were studied. Both diffuse systemic sclerosis and limited systemic sclerosis patients were included in this study. Detailed clinical examination, basic blood investigations, immunological tests, ECG, $\mathrm{x}$ ray chest, echocardiography, HRCT chest and upper gastrointestinal scopy were done in all patients.

\section{RESULTS}

3 patients (12\%) with LcSS had ECG abnormalities. One patient with DcSS (4\%) had ECG abnormality. The ECG abnormalities were sinus tachycardia, VPC (1 pt), T-wave inversion in inferior leads (1 pt) and LAHB with left axis deviation (1 pt). Among the total 25 patients, 6 patients (24\%) had abnormalities in echocardiography. 4 patients with LcSS and remaining 2 (34\%) with DcSS. The common echo abnormalities were pulmonary hypertension with dilatation of right atrium and right ventricle (84\%). Pulmonary hypertension was secondary to interstitial lung diseases.

\section{CONCLUSION}

In this study, we found that $12 \%$ of LcSS had ECG abnormalities. Echo abnormality was seen in $66 \%$ of LcSS and $34 \%$ of DcSS. In our study, we found that cardiac rhythm disturbances occurred even in asymptomatic individuals.

\section{KEYWORDS}

Raynaud's Phenomenon, Contraction Band Necrosis, Cardiac Rhythm Disturbances.

HOW TO CITE THIS ARTICLE: Saravanan M, Nagaventhan M, Arivudainambi P, et al. Study on cardiac involvement in systemic sclerosis. J. Evolution Med. Dent. Sci. 2018;7(28):3188-3191, DOI: 10.14260/jemds/2018/717

\section{BACKGROUND}

Systemic sclerosis is a generalised disorder of connective tissue characterised by diffuse vascular lesions, fibrosis of skin and major organ systems involvement including cardiac, pulmonary and gastrointestinal system. The Raynaud's phenomenon and vasculopathy are important pathological hallmarks of systemic sclerosis. $1,2,3$ The above pathological features affect the cardiovascular system, especially conduction pathway of electrical impulse which manifests as abnormal arrhythmia on ECG.4,5 It also involves cardiac valves, pericardium, myocardium and coronary vessels. 4,5 The aim of this descriptive study is to assess the cardiac involvement in patients with systemic sclerosis.

\section{MATERIALS AND METHODS}

Detailed clinical examination, basic blood investigations, immunological tests, ECG, x-ray chest, echocardiography,

'Financial or Other Competing Interest': None.

Submission 08-05-2018, Peer Review 23-06-2018,

Acceptance 01-07-2018, Published 09-07-2018.

Corresponding Author:

Dr. Mayilsamy Saravanan,

\#116, $4^{\text {th }}$ Cross, Fathima Nagar,

Woraiyur, Tiruchy-620003,

Tamilnadu, India.

E-mail:dmrsaravanan@gmail.com

DOI: $10.14260 /$ jemds/2018/717
HRCT chest and upper gastrointestinal scopy were done in all patients.

\section{Material and Selection}

This study was conducted in the Department of Rheumatology, KAP Viswanatham Govt. Medical College, Trichy, Tamilnadu from the period of August 2017 - April 2018. 25 patients of systemic sclerosis were studied. The study was conducted after obtaining approval from Institutional Ethical Committee. The written consent was obtained from all patients who were participated in the study.

\section{Sample Size}

25

\section{Study Design \\ Descriptive study.}

\section{Inclusion Criteria}

Both diffuse systemic sclerosis and limited systemic sclerosis patients were included in this study. Localised scleroderma and overlapping syndrome patients were not included in this study.

The sample size of $25.23(92 \%)$ were female and $2(8 \%)$ were males of 23 (92\%) who had Limited Cutaneous Systemic Sclerosis (LcSS) and 2 (8\%) had Diffuse Cutaneous 
Systemic Sclerosis (DcSS). Age: 15 - 55 years' duration of disease- 8 months - 5 years.

\section{Statistical Analysis}

Statistical analysis was performed by using SPSS software 22 . Mean, mode and median was measured for numerical values. The Chi-square test was used to find the association of type of systemic sclerosis and ECG abnormality.

\section{RESULTS}

3 patients $(12 \%)$ with LcSS had ECG abnormalities. One patient with DcSS (4\%) had ECG abnormality. The ECG abnormalities were Sinus tachycardia, VPC (1 pt), T-wave inversion in inferior leads (1 pt), LAHB with left axis deviation (1 pt). Among the total 25 patients, 6 patients (24\%) had abnormalities on echocardiography. 4 patients were LcSS and remaining 2 (34\%) were DcSS.

The common echo abnormalities were pulmonary hypertension with dilatation of right atrium and right ventricle (84\%). Pulmonary hypertension was secondary to interstitial lung diseases. Dilated cardiomyopathy with left ventricle apical thrombus was in one patient (16\%). 3 patients had trivial MR (50\%). 3 patients had trivial TR (50\%). 1 patient (16\%) had mild pericardial effusion. 12 (48\%) LcSS and 2 (8\%) DcSS patients had interstitial lung diseases proven by HRCT. 7 patients (28\%) had lax lower oesophageal sphincter in upper gastrointestinal scopy. 3 patients (12\%) had hiatus hernia. 2 patients (8\%) had cricopharyngeal web.

\begin{tabular}{|c|c|c|}
\hline Sl. No. & Type of Cardiac Involvement & No. of Patients \\
\hline 1 & $\begin{array}{c}\text { Pulmonary hypertension with } \\
\text { dilation of right atrium and right } \\
\text { ventricle }\end{array}$ & 4 \\
\hline 2 & $\begin{array}{c}\text { Ventricular premature contraction } \\
\text { in ECG }\end{array}$ & 1 \\
\hline 3 & Left anterior hemiblock in ECG & 1 \\
\hline 4 & T-wave inversion in ECG & 1 \\
\hline 5 & Dilated cardiomyopathy & 1 \\
\hline 6 & Trivial MR & 3 \\
\hline 7 & Trivial TR & 3 \\
\hline 8 & Pericardial effusion & 1 \\
\hline \multicolumn{2}{|c|}{$\begin{array}{c}\text { Table 1. Various Manifestations of Cardiac } \\
\text { Involvement in our Study Group }\end{array}$} \\
\hline \multicolumn{2}{|c|}{} \\
\hline
\end{tabular}

\begin{tabular}{|c|c|c|c|c|}
\hline & $\begin{array}{c}\text { Diffuse } \\
\text { Systemic } \\
\text { Sclerosis }\end{array}$ & \begin{tabular}{|c|} 
Limited \\
Cutaneous \\
Sclerosis \\
\end{tabular} & Total \\
\hline \multirow{2}{*}{$\begin{array}{c}\text { ECG } \\
\text { Changes }\end{array}$} & Absent & 1 & 20 & 21 \\
\hline & Present & 1 & 3 & 4 \\
\hline \multicolumn{2}{|c|}{ Total } & 2 & 23 & 25 \\
\hline
\end{tabular}

$\mathrm{P}$-value 0.171495 . The result was not significant at $\mathrm{p}<0.05$.

\section{DISCUSSION}

Cardiac involvement in systemic sclerosis is divided into direct cardiac involvement and indirect effect in heart by other organ involvement like pulmonary hypertension, interstitial lung disease and scleroderma renal crisis.

Systemic sclerosis can involve almost all structures of cardiovascular system like conduction pathway, heart valves, endocardium, myocardium, pericardium, coronary arteries and pulmonary arteries.6,7

The clinical manifestations of cardiac involvement are cardiac myositis, cardiac failure, coronary artery heart disease, conduction system disturbances and pericardial disease. The symptoms of cardiac involvement are chest pain, dyspnoea, palpitation and syncope. Systemic sclerosis patients with chest pain, which can mimic like angina caused by ischaemic heart disease. 6,7

The symptoms of cardiac involvement in systemic sclerosis may be under-estimated, because of occult nature of symptoms and signs. Sometimes, the symptoms of cardiac involvement may be attributed to musculoskeletal involvement, pulmonary involvement and oesophageal involvement.

Systemic sclerosis patients with right heart failure usually present with progressive shortness of breathing, ascites, pedal oedema and congestive hepatomegaly. In our study, no patients had signs of severe right heart failure.

Patients with arrhythmia usually presented with palpitation and syncope. The higher risk findings in systemic sclerosis are presence of cardiac failure, poor right ventricular function, pulmonary hypertension, high right atrial pressure and documented right ventricular arrthymias. 6,7 In systemic sclerosis, cardiac involvement has occurred after three years of skin involvement.

Pulmonary hypertension in systemic sclerosis has impact over the cardiac function. Pulmonary hypertension is defined as elevation of mean pulmonary artery pressure $>25 \mathrm{mmHg}$ at rest, during exercise of more than $30 \mathrm{mmHg}$ and during echo measurement pulmonary arterial systolic pressure of more than $40 \mathrm{mmHg}$. Right atrial area index and pericardial effusion indices usually reflect profound right heart failure. High mean right atrial pressure is consistently associated with poor survival.

84 percentage of our patients had dilation of right atrium and right ventricle, which were detected by echocardiography. Systemic sclerosis patients should undergo echocardiography yearly for periodic screening.

TAPSE [Tricuspid Annular Plane Systolic Excursion] has been correlated with mortality in patients of systemic sclerosis with pulmonary hypertension and idiopathic pulmonary hypertension.

In patients with severe pulmonary hypertension syncope, sudden cardiac death can occur as a result of arrhythmia and severe right heart failure.

The outcome of idiopathic pulmonary hypertension is better than connective tissue associated with pulmonary hypertension. Digital ulcers and telangiectasia are associated with pulmonary hypertension.

Pericardial effusion can be frequently associated with pulmonary hypertension. If inflammation is the cause of pericardial effusion, immunosuppressive drugs can improve the condition.

Pericardial effusion with cardiac failure can be reduced by diuresis. In our study only one patient had pericardial effusion, which was mild effusion. Percutaneous drainage should be avoided in patients with pericardial effusion with severe pulmonary hypertension. This procedure may cause haemodynamic compromise and cardiac death. In our study, no patients had both pulmonary hypertension and pericardial effusion at the same time. 
Nodular thickening of mitral valve, aortic valve, shortening of chordae tendineae and vegetations in cardiac valves were noted in some autopsy studied. In our study, no patients had nodular thickening of valves.

Echocardiography is a useful non-invasive technique to assess the severity of cardiac involvement. Pulmonary artery hypertension is confirmed by right atrial catheterisation. It was not done in our study.

Pulmonary function test in pulmonary hypertension reveal reduced DLCO. It was not done in our study. Pulmonary hypertension precedes skin hardening in limited systemic sclerosis patients.

The risk factors of pulmonary hypertension are limited systemic sclerosis with anti-centromere antibody, severe Raynaud's phenomenon, disease start at late age and presence of antibodies to U1RNP, U3RNP (Fibrillarin) and B23. Antibodies to U1RNP, U3RNP (Fibrillarin) and B23 were not done in our study.

In systemic sclerosis, pulmonary hypertension results from interstitial pulmonary artery disease and interstitial lung disease. In our study, all the pulmonary artery hypertensive patients had interstitial lung disease also.

Autoimmunity, vasculopathy and fibrosis are the triad of pathogenesis of systemic sclerosis. Vasculopathy is one of the aetiopathogenesis of macrovascular disease in systemic sclerosis. Raynaud's phenomenon may be the initial manifestation of systemic sclerosis. 90 to 98 percentage of systemic sclerosis patients have Raynaud's phenomenon as the initial manifestation. $8,9,10$

Raynaud's phenomenon is included in ACR-EULAR criteria for systemic sclerosis. Because of cardiac Raynaud's phenomenon, contraction band necrosis occurred which was due to ischaemia-reperfusion injury along with electrical impulse conduction pathway.8,9 Myocardial fibrosis is also a part of cardiac pathogenesis by systemic sclerosis.

Contraction band necrosis can cause sinoatrial block, first degree AV nodal block, second degree heart block, right bundle branch block and left bundle branch block. In our study the ECG abnormalities were sinus tachycardia, T-wave inversion in inferior leads, left anterior hemiblock with left axis deviation and ventricular premature contraction.

Thallium scan is used to assess the myocardial perfusion. Calcium channel blockers, ACE inhibitors, Phospho 5 esterase inhibitors and Endothelin receptor blockers are used in systemic sclerosis patients to improve the myocardial perfusion. Thallium scan is used to monitor the efficacy of above-mentioned drugs.

Studies about myocardial perfusion was assessed by single photon emission computed tomography scans which showed half of the systemic sclerosis patients had impairment of left ventricle relaxation. ${ }^{10}$ Cardiac MRI can find oedema in myocardium and myocardial fibrosis. In our study, patients did not undergo both cardiac MRI and Thallium scan because of financial constraint.

Patchy myocardial fibrosis is characteristic finding in systemic sclerosis. Myocardial fibrosis can cause left ventricular hypertrophy, which lead to both systolic and diastolic dysfunction. Left ventricle systolic dysfunction is not uncommon in systemic sclerosis. ${ }^{10}$ In our study, none of the patients had left ventricular hypertrophy during echocardiographic measurement.
Myocardial fibrosis is the aetiology for atrial tachyarrhythmias and ventricular tachyarrhythmias in systemic sclerosis patients. Fibrotic process in heart may be secondary to chronic inflammation.

Pro-brain natriuretic peptide is a marker for cardiac involvement. Stretching of atrial and ventricle can cause release of Pro-brain natriuretic peptide. The upper limit of Pro-brain natriuretic peptide is $125 \mathrm{pg} / \mathrm{mL}$. It was not measured in our study.

Systemic sclerosis patients with elevated creatine kinase who have associated with cardiac dysfunction, congestive cardiac failure and cardiac death. ${ }^{10}$ Sometimes, creatine kinase level may be normal even in the presence of cardiac inflammation. Creatine kinase was not measured in our study.

Heart irregularities are the earliest symptom of systemic sclerosis. In our study ECG abnormalities like sinus bradycardia, ventricular premature contraction, T-wave inversion and left anterior hemiblock occurred in our patients who did not have any symptoms regarding cardiovascular system involvement.

24 hours Holter ECG monitoring is useful to detect conduction disturbances in systemic sclerosis patients who were with symptoms or without symptoms.

Anecdotally, systemic sclerosis patients with reduced ejection fraction with normal coronary arteries, normal left ventricle size may be improved by immunosuppressive drugs. Left ventricle diastolic dysfunction correlates with disease duration and are associated with severity of cutaneous disease. 10

Pericardial involvement in systemic sclerosis are fibrinous pericarditis, pericardial adhesions and pericardial effusion. Large pericardial effusion with cardiac tamponade, which can occur even before skin thickening and diagnosis of scleroderma.10,11 In our study, none of the patients had large pericardial effusion with cardiac tamponade.

In our study, sinus tachycardia occurred in one symptomatic patient who had chest pain. Coronary angiography was done for that patient which did not reveal any occlusion in coronary vessels. Generally, epicardial coronary arteries are angiographically normal in systemic sclerosis patients.10,11 Systemic sclerosis patients usually have significant peripheral artery narrowing than myocardial vessels.

HRCT chest was done for all patients in our study, since interstitial lung disease is one of the causes for pulmonary hypertension. ${ }^{10,11}$

\section{Limitation of Study}

- Small sample size.

- Short study period.

- Holter ECG monitoring was not done.

\section{CONCLUSION}

In this study, we found that $12 \%$ of LcSS had ECG abnormalities. Echo abnormality was seen in $66 \%$ of LcSS and $34 \%$ of DcSS. In our study, we found that cardiac rhythm disturbances occurred even in asymptomatic individuals. Early evaluation of cardiac involvement and pulmonary involvement in systemic sclerosis patients can significantly reduce the morbidity. 


\section{REFERENCES}

[1] Harrick AL. Pathogenesis of Raynaud's phenomenon. Rheumatology (Oxford) 2005;44(5):587-96.

[2] Carpentier PH, Satger B, Poensin D, et al. Incidence and natural history of Raynaud's phenomenon: a longterm follow-up (14 years) of a random sample from the general population. J Vasc Surgery 2006;44(5):1023-8.

[3] Ho M, Veale D, Eastmond C, et al. Microvascular disease and systemic sclerosis. Ann Rheum Dis 2000;59(1):39-43.

[4] Follansbee WP, Zerbe TR, Medsger TA Jr. Cardiac and skeletal muscle disease in systemic sclerosis (scleroderma): a high risk association. Am Heart Journal 1993;125(1):194-203.

[5] Murata I, Takenaka K, Shinohara S, et al. Diversity of myocardial involvement in systemic sclerosis: an 8year study of 95 Japanese patients. Am Heart Journal 1998;135(6 Pt 1):960-9.

[6] Satoh M, Tokuhira M, Hama N, et al. Massive pericardial effusion in scleroderma: a review of five cases. Br J Rheumatology 1995;34(6):564-7.
[7] Plazak W, Zabinska-Plazak E, Wojas-Pelc A, et al. Heart structure and function in systemic sclerosis. Eur J Dermatology 2002;12(3):257-62.

[8] Candell-Riera J, Armadans-Gil L, Simeon CP, et al. Comprehensive non-invasive assessment of cardiac involvement in limited systemic sclerosis. Arthritis Rheum 1996;39(7):1138-45.

[9] Alexander EL, Firestein GS, Weiss JL, et al. Reversible cold-induced abnormalities in myocardial perfusion and function in systemic sclerosis. Ann Intern Med 1986;105(5):661-8.

[10] Harrison NK, Glanville AR, Strickland B, et al. Pulmonary involvement in systemic sclerosis: the detection of early changes by thin section CT scan, bronchoalveolar lavage and $99 \mathrm{mTc}$-DTPA clearance. Respir Med 1989;83(5):403-14.

[11] Champian HC. Systemic sclerosis. Rheumatic Disease Clinics of North America 2014. 